\title{
A Construction Supply Chain Management Process with RFID/WSN-based Logistics Equipment
}

\author{
Tae-Hong Shin ${ }^{1}$, Su-Won Yoon ${ }^{2 *}$, and Sangyoon Chin $^{3}$
}

Received June 18, 2012 / Revised August 3, 2012 / Accepted October 12, 2012

\begin{abstract}
Construction supply chain management (CSCM) has become one of the critical factors that determine the success of a construction project as it becomes increasingly complicated and mega-sized. Particularly for high-rise or mega-sized building construction, just-in-time supply chain management is required due to lack of storage space and effective logistics for construction components and materials at a construction site. Despite the fact that research and development of radio frequency identification (RFID) and wireless sensor network (WSN) technology have been performed, construction project managers still need to carry mobile devices and check material and component flow at each stage of the supply chain process. This research proposes that the equipment used in the construction supply chain process, such as movers, trailers, gates, and hoists, can become main actors in the supply chain process using RFID and WSN technologies. And the proposed equipment and process focused on a solution to the redundancy identification problem, which has been observed in operations that use RFID/WSN-based processes for construction logistics. This paper also presents issues identified through verification and validation of the research results and proposes further studies
\end{abstract}

Keywords: Radio frequency identification (RFID); Wireless sensor network (WSN); ZigBee; Redundancy identification; Construction supply chain management; RFD/WSN-based equipment; RFID/WSN-based communication; Process automation; Real-time logistics information sharing

\section{INTRODUCTION}

Construction supply chain management (CSCM), a process of monitoring and controlling material and information flow, increasingly involves more and more types and quantities of construction components. And just-in-time-based CSCM is required due to limited space of construction sites as construction projects become larger and higher. Therefore, more accurate and sophisticated information management is necessary in the order, manufacture, production, storage, and erection processes of construction components, particularly in high-rise and mega-sized construction projects that have many types and quantities of materials.

The emergence of RFID/WSN technologies has invoked various research and development in an effort to resolve the CSCM needs mentioned above, and they have shown opportunities for and the feasibility of RFID/WSN-based applications in the construction industry (Chin et al. 2008; Song et al. 2004; Ergen et al. 2007; Yoon et al. 2005, Chin et al. 2007; Jaselskis et al., 2003; Song et al., 2004; Yoon et al., 2011).

However, existing research (Figure I) has the limitation of requiring human involvement at every step of the supply chain to monitor component flow. That is, managers need to carry mobile devices to collect data, or a stationary device needs to be installed at a fixed location through which the components pass. Mobile device use time is limited at construction sites as well due to battery capacity, e.g., a $900 \mathrm{MHz}$ personal data assistant-based RFID reader can be continuously used for about 2 hours.

The use of stationary RFID readers at various locations in the construction supply chain process have more numerous barriers than the use of mobile devices since stationary RFID readers require more readers to expand the reading range, which increases cost, results in redundant reading by more than one reader, can allow the reading of unnecessary items that are not yet in the supply chain process, and lack the safety, convenience, and mobility required for use at construction sites.

This research proposes that the equipment involved in the supply chain process, such as movers, trailers, gates, and hoists, can become main actors that collect and share information with other actors(e.g. human, material etc.) in the process using RFID and WSN technologies (Figure I).

As shown in Figure I, this research differs from existing studies (Chin et al. 2008; Ergen et al. 2007; Song et al. 2006; Jaselskis et al., 1995; Jaselskis et al., 2003; Han et al. 2006; Cho et al. 2011, Chin et al. 2008) in that it examined a structure that identifies RFID materials when they are shipped and then transmits the material information using the WSN technology along the supply chain management process. No additional process is required to confirm whether they are RFID-based material in every process of the supply chain management system.

\footnotetext{
${ }^{1}$ Senior Consultant, Ph. D., Samsung SDS, Yoeksam-dong, Gangnam-gu, Seoul 135-918, Republic of Korea, taehong.shin@ samsung.com

${ }^{2}$ Section Manager, Ph. D.,R\&D Center, POSCO E\&C, Songdo-dong, Yeonsu-gu, Incheon 406-840, Republic of Korea, yoonsuwon@ poscoenc.com (*Corresponding Author)

3 Professor, Department of Civil, Architectural, and Environmental System Engineering, Sungkyunkwan University, Cheoncheon-dong, Jangan-gu, Suwon 400-746, Republic of Korea, e-mail address
} 


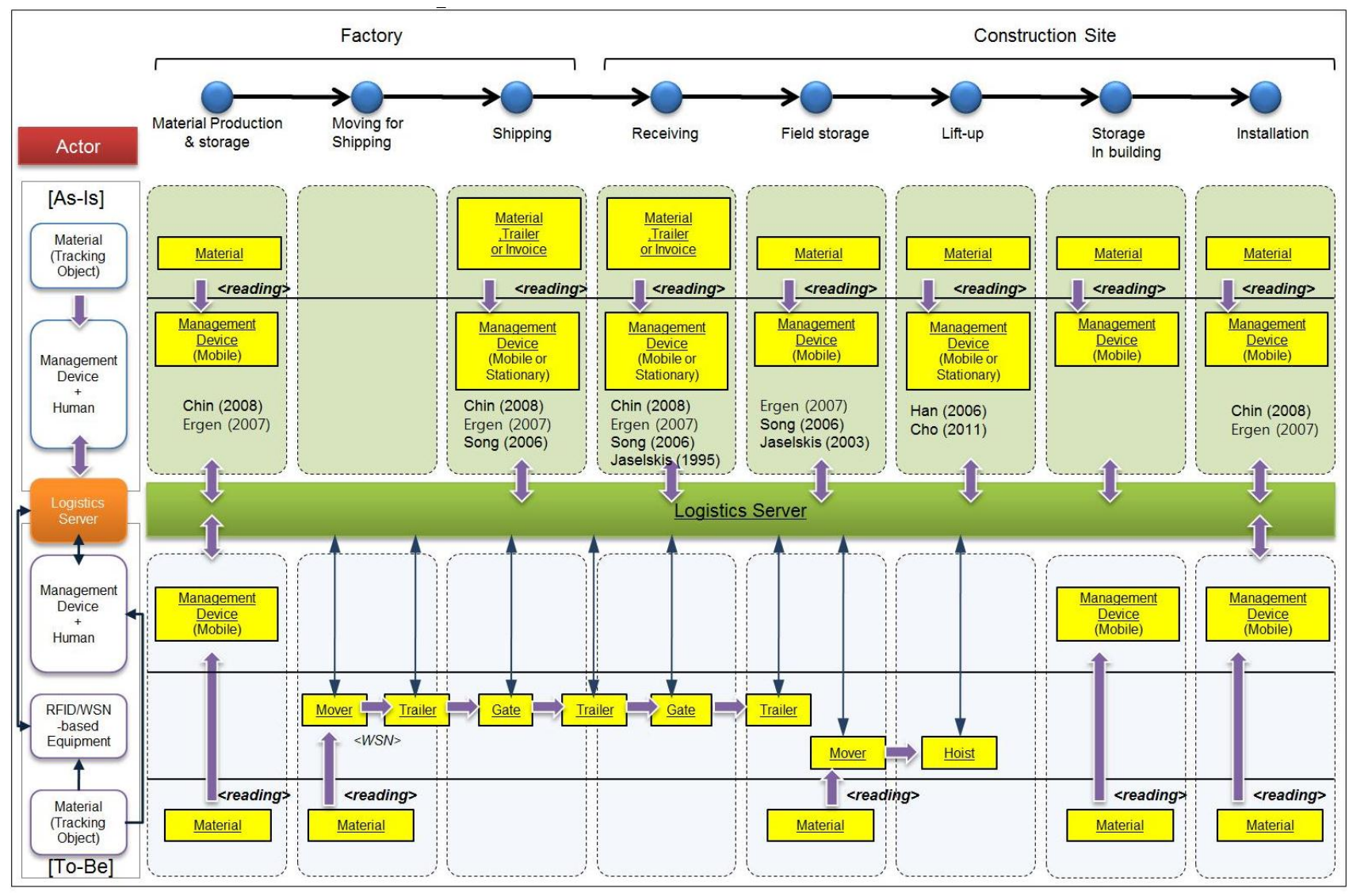

FIGURE I

ACTOR RELATIONSHIP IN THE RFID/WSN-BASED CSCM ENVIRONMENT

Therefore, the objective of this research is to build an effective construction supply chain process framework by transforming the equipment mentioned above into active actors that can recognize materials and components and can communicate and share the collected information with others, including logistics servers. Field tests were performed to verify and validate the framework proposed by this research. Further research and issues identified during the field tests are described and discussed below.

\section{RESEARCH SCOPE AND METHOD}

This research focuses on the CSCM process that spans from the material order to the production, delivery, and erection at a construction site and the monitoring and controlling of the flow of material/components. This research considers the equipment involved in the logistics main actors in CSCM. Although there are various kinds of CSCM-related equipment, four kinds of equipment were selected and transformed as main actors using RFID/WSN technologies: 1) a mover; 2) a trailer that horizontally transports material and components; 3) a gate that can monitor the passage of material/components; and 4) a hoist that can vertically move materials and components.

The overall procedures of this research are as follows:
(1) Literature related in RFID/WSN and CSCM were reviewed with focus on the applications in the construction industry.

(2) RFID/WSN-based CSCM processes are developed with the assumption that logistics equipment can become main actors in a RFID/WSN-based CSCM process.

(3) Using RFID/WSN technologies, toolkits were developed to transform logistics equipment into actors that can collect, communicate, and share CSCM information with other actors, such as other equipment, managers, and logistics servers.

(4) The RFID/WSN-based CSCM process along with toolkits and equipment were verified and validated using field tests to show the process feasibility.

(5) Finally, issues during field tests were identified. These issues are discussed in this paper along with future research topics.

\section{RFID/WSN-BASED CSCM-RELATED LITERATURE REVIEW}

\section{A. RFID Technology in Construction}

RFID is considered next-generation barcode technology that is able to overcome the limits of present barcode technology including low readability under direct sunlight and lack of durability in harsh construction conditions (Jaselskis and El-Misalami, 2003). Taking 
advantage of the merits of RFID, many research projects have studied its application in the construction industry.

The potential to apply RFID in various processes, such as concrete operation from ordering to testing at the jobsite, cost coding of labor and equipment, and material control, has been demonstrated (Jaselskis et al., 1995; Jaselskis et al., 2003). A flowchart to support the RFID technology selection was developed through consideration of the various conditions and factors in the construction industry (Jaselskis and El-Misalami, 2003).

Research on RFID applications has been performed in the areas of material receipt at power plants and refinery projects (Jaselskis et al., 2003), field trials for prefabricated pipe spools (Song et al., 2006), tool tracking (Goodrum et al., 2006), and structural steel member tracking (Chin et al., 2008).

Although RFID technology is becoming increasingly advanced and its price is decreasing, current RFID technology has various barriers to its application in the construction industry as identified by existing research projects. These barriers include a lack of standardization, potential interference from metal objects and other radio frequency (RF) signals, closed system environments for RFID technology, practitioners' skepticism toward RFID, and high hardware costs (Jaselskis et al., 1995; Jaselskis et al., 2003; Goodrum et al., 2006).

Furthermore, there has been a lack of research on the practical application of RFID technology in the construction industry; that is, specifications of RFID technology, such as reading distance and readability, can differ in practical use in construction projects. In addition, because RFID tags can be applied differently even in the same construction project depending on the process to which they are applied, it is necessary to pursue effective and efficient application methods that can improve existing processes by adopting RFID while considering the technological economy.

\section{B. WSN Technology in Construction}

WSN is a communication technology that helps send and receive information using (1) communication protocols between objects that try to share information and (2) the information collected by RFID tag reading (Akyildiz et al., 2002).

WSN technologies were recently divided into the following: wide area network (WAN), metropolitan area network (MAN), local area network (LAN), and personal area network (PAN). According to a literature review, the practical use of WSN in construction sites is limited. Considering power consumption-related technical quality, PAN, such as Bluetooth and ultra-wideband (UWB), ZigBee, and Wi-Fi, is considerable to adapt in construction industry (Behzadan et al., 2008; Lu et al., 2007; Jang et al., 2008 etc.).

Bluetooth (IEEE 802.15.1) is a short-range wireless connection technology that connects computers, printers, cellular phones, and a variety of digital appliances in home and office settings using the wireless frequency of 2.4 GHz. Although it offers high data transmitting speed, its electric power waste level is high and the maximum number of simultaneously connected machines is restricted.

UWB (802.15.3a) can facilitate the rapid transfer of huge amounts of data through an ultra-high frequency at a short distance of approximately $10 \mathrm{~m}$ using very little power (a quarter of Bluetooth). Compared with Bluetooth, this technology will be utilized to quickly transmit relatively considerable amounts of data. However, the commercial use of UWB is delayed due to differences in electric waves, the limitation of transferring fundamental technology, and the problem of standardization between industries. There is also a limit related to transmission distance and power consumption. It is difficult to widely adapt to construction sites due to the short distance abilities of wireless transmission and the rapid battery consumption.

ZigBee (IEEE 802.15.4) technology is attracting attention as the new international standard related to short distance communication in wireless network and recent noteworthy ubiquitous computing. Although its data transmission amount is insufficient, it has a bidirectional wireless network and ensures a long battery life ranging from several months to years. ZigBee (IEEE 802.15.4) technology is expected to provide economic wireless data communication solutions.

With regard to the construction industry, this research adopted ZigBee technology as the wireless data communication protocol for inter-equipment communication. ZigBee technology is structurally simple, and the cost of its implementation is less than that of its competitors. This technology allows more devices to be connected to one network and consumes less power, prolonging battery life. Finally, the network is configurable in various ways, including peer-to-peer (P2P), star, and mesh (Andrew, 2007).

The ZigBee module with the P2P network configuration was attached to WSN equipment and played the role of a peer, meaning that a coordinator was not required for inter-equipment communication. Meanwhile, wireless broadband (WiBro) was adopted to support communication between intelligent equipment and the CMS. WiBro is a wireless broadband technology for IEEE 802.16e that is commercially available in South Korea through portable Internet connections with rates of around 30-50 Mbit/s (Lee et al., 2006).

ZigBee technology has been adopted in constructionrelated research and development with a main focus on inter-equipment communication, such as ZigBee-based near-miss accidents at construction sites (Wu et al., 2010), ZigBee-based logistics pallet location tracking (Haifeng et al., 2008), localization technique RF + ultrasound sensor interface (Skibniewski et al., 2007), ZigBee-based intervehicle communication (Zhang et al., 2007), and ZigBee application on intelligent bus dispatch (Guizhen et al., 2007). Meanwhile, other wireless sensor network technologies have been adopted in construction-related areas as well with a focus on positioning and tracking, such as construction indoor asset tracking (Cho et al., 2010), user localization in the indoor construction environment (Khoury et al., 2009), construction vehicle positioning and tracking ( $\mathrm{Lu}$ et al., 2007), location tracking for context- 
specific information delivery (Behzadan et al., 2008), building environmental monitoring (Jang et al., 2008), and structural health monitoring (Hoult et al., 2010).

\section{Actor Relationship R\&D in RFID/WSN-Based CSCM}

The construction industry RFID- and WSN-based research projects mentioned above can be categorized into three types of CSCM actor relationships (Table I): 1) Human-Material-RFID tag, 2) Human-Material-RFID tag-WSN, and 3) Human-Material-RFID tag-WSNEquipment for Logistics.

Existing research projects belong to categories 1) and 2 ), whereas it is the research in category 3) in which logistics equipments are defined as main actors to collect, communicate, and share CSCM information and a new concept of RFID/WSN-based CSCM process is proposed. Related to this research, parts of the research concept and results have been published that describe the following: the concept of the RFID/WSN-based CSCM process (Shin et al., 2011); RFID/WSN-based equipments such as movers and trailers (Shin et al., 2011), gate sensors (Yoon et al., 2011), and hoists (Cho et al., 2011); and serviceoriented architecture (SOA)-based environments for RFID/WSN-based CSCM (Shin et al., 2011).

Shin et al. (2011) showed that SOA could effectively improve logistics-related information management efficiency in the RFID/WSN-based heterogeneous CSCM environment. Yoon et al. (2011) and Cho et al. (2011) showed that the information collected from RFID tag reading can be shared using WSN technology through a recognition test of RFID/WSN-based gates and hoists. However, these papers do not show or propose the full process of CSCM in which RFID/WSN-based equipment is utilized, nor do they show what kind of roles RFID/WSN-based equipment can have in the CSCM process. Therefore, this paper aims to shed new light on RFID/WSN-based equipment and service-based logistics information management environments from the aspect of the full CSCM process and proposes that use of the RFID/WSN-based equipment can improve the CSCM process by having them participate in the process.

\section{DEVELOPMENT OF RFID/WSN-BASED CONSTRUCTION SUPPLY CHAIN MANAGEMENT}

\section{A. Motivation of Research}

Since 2006, the authors have conducted a research project called Next Generation Intelligent Construction Supply Chain Management (NICS), the aim of which is to develop a process and system that support proactive construction supply chain management in real time by utilizing WSN and RFID technologies. Such a system could then be used to solve problems associated with technology evolution, errors made in the collection of information, differences in the levels of information shown by project-participating parties, and additional work that may become necessary to complete the gathering of information (Figure II).
RFID/WSN-based CSCM Environment

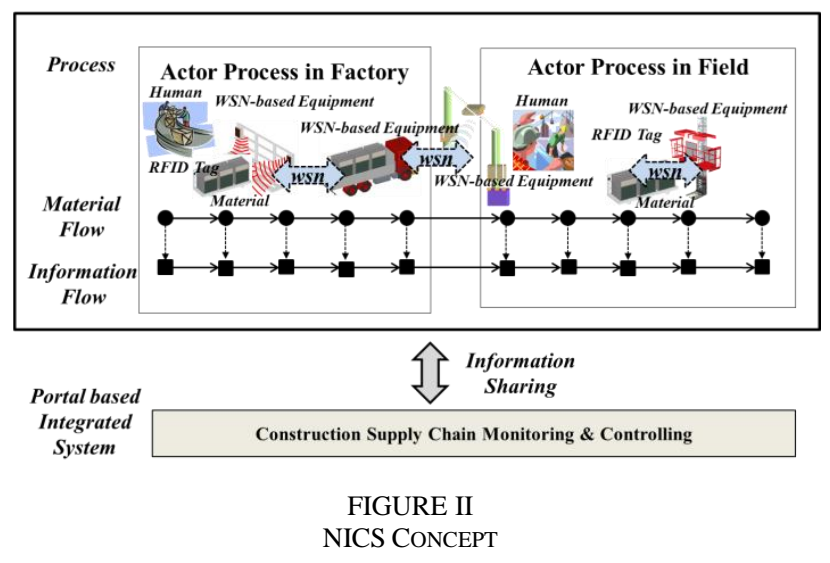

In this project, "next generation" refers to the use of RFID/WSN technology to improve the current CSCM process, and the term "intelligent" refers to equipment such as pallets, trailers, hoists, and gates that is able to recognize the components or materials that they possess and can then communicate their information to other equipment or actors involved via RFID/WSN. In other words, the NICS project is an initiative that will be used to establish an intelligent CSCM environment in which equipment used to be regarded as a mere vehicle for moving materials from place to place is endowed with intelligence. With this intelligence, the equipment can then recognize material information and transmit and share this information with other equipment via wireless networks.

As shown in Figure II, consider a construction material such as plaster boards that is manufactured and boxed at a factory. An RFID tag is attached to the box of boards and the intelligent pallet is given the name of the material to allow for the release of the item. The pallet then reads the RFID tag and automatically recognizes the information. To release the material for which information has been loaded into the pallet, it is first delivered to an intelligent trailer. ZigBee communication then enables the recognition of the materials that have been loaded onto the transportation vehicles and verifies whether the correct items have been loaded for delivery. When the intelligent trailer approaches GateSensor (Yoon et al., 2011), the device will determine whether the planned materials are being released as scheduled.

To ensure the establishment of a proper environment, a number of tasks must first be performed, including the development of an RFID/WSN-based CSCM process (Shin et al., 2011), equipment (Intelligent Mover [IM; Shin et al., 2011], Intelligent Trailer [IT; Shin et al., 2011], GateSensor, Intelligent Hoist [IH; Cho et al., 2011]), systems, and various interfaces (Shin et al. 2011) between equipment, equipment and servers, or between servers and the existing legacy system. 
TABLE I

RFID/WSN-BASED CSCM PROCESS AND ACTOR RELATIONSHIP

\begin{tabular}{|c|c|c|c|c|c|c|c|c|}
\hline \multirow{2}{*}{$\begin{array}{l}\text { Headquarter/ } \\
\text { Factory/ } \\
\text { Field }\end{array}$} & \multirow[b]{2}{*}{ No } & \multirow[b]{2}{*}{ Process } & \multicolumn{6}{|c|}{ Actor } \\
\hline & & & Human & Material & RFID Tag & $\begin{array}{l}\text { RFID/WSN } \\
\text { Equipment }\end{array}$ & $\begin{array}{l}\text { Management } \\
\text { Device }\end{array}$ & $\begin{array}{l}\text { ERP/ } \\
\text { MRP }\end{array}$ \\
\hline \multirow{2}{*}{$\begin{array}{c}\text { Head } \\
\text { quarter }\end{array}$} & $\mathrm{P} 01$ & Material Req. Planning & $\sqrt{ }$ & & & & & \\
\hline & $\mathrm{P} 02$ & Manufacturing Planning & $\sqrt{ }$ & & & & & $\sqrt{ }$ \\
\hline \multirow{8}{*}{ Factory } & $\mathrm{P} 03$ & $\begin{array}{l}\text { Production and Quality } \\
\text { Check }\end{array}$ & $\sqrt{ }$ & & & & & $\sqrt{ }$ \\
\hline & P04 & Factory Stock & $\sqrt{ }$ & $\sqrt{ }$ & & & & \\
\hline & $\mathrm{P} 05$ & RFID tagging to Material & $\sqrt{ }$ & $\sqrt{ }$ & $\sqrt{ }$ & & & \\
\hline & P06 & Loading Material to IM & $\sqrt{ }$ & $\sqrt{ }$ & $\sqrt{ }$ & $\sqrt{ }$ & & \\
\hline & P07 & IM-IT Communication & & $\sqrt{ }$ & $\sqrt{ }$ & $\sqrt{ }$ & & \\
\hline & $\mathrm{P} 08$ & Inspection & $\sqrt{ }$ & $\sqrt{ }$ & $\sqrt{ }$ & & $\sqrt{ }$ & \\
\hline & P09 & Issue e-Invoice & $\sqrt{ }$ & & & & $\sqrt{ }$ & \\
\hline & $\mathrm{P} 10$ & IT-GS Comm. at Factory & & $\sqrt{ }$ & $\sqrt{ }$ & $\sqrt{ }$ & & \\
\hline \multirow{10}{*}{ Field } & P11 & IT-GS Comm. at Gate & & $\sqrt{ }$ & $\sqrt{ }$ & $\sqrt{ }$ & & \\
\hline & $\mathrm{P} 12$ & Receipt Confirmation & $\sqrt{ }$ & & & & $\sqrt{ }$ & \\
\hline & $\mathrm{P} 13$ & Field Stock & $\sqrt{ }$ & & & & & \\
\hline & $\mathrm{P} 14$ & Loading Material to IM & $\sqrt{ }$ & $\sqrt{ }$ & $\sqrt{ }$ & $\sqrt{ }$ & & \\
\hline & $\mathrm{P} 15$ & IM to IH & $\sqrt{ }$ & $\sqrt{ }$ & $\sqrt{ }$ & $\sqrt{ }$ & & \\
\hline & $\mathrm{P} 16$ & IM-IH Communication & & & & $\sqrt{ }$ & & \\
\hline & $\mathrm{P} 17$ & Loading IM to IH & $\sqrt{ }$ & $\sqrt{ }$ & $\sqrt{ }$ & $\sqrt{ }$ & & \\
\hline & P18 & $\begin{array}{l}\text { Unloading IM on the } \\
\text { Planned Floor }\end{array}$ & $\sqrt{ }$ & $\sqrt{ }$ & $\sqrt{ }$ & $\sqrt{ }$ & & \\
\hline & P19 & Installation & $\sqrt{ }$ & $\sqrt{ }$ & & & & \\
\hline & $\mathrm{P} 20$ & Final Inspection & $\sqrt{ }$ & & & & $\sqrt{ }$ & \\
\hline
\end{tabular}

\section{B. RFID/WSN-Based CSCM Process and Actors}

This research proposes that RFID/WSN-based logistics equipment can become main process actors. As shown in Table I, seven main actors, such as humans, materials, RFID tags, RFID/WSN-based equipment, management devices, and ERP/MRP, are involved in the RFID/WSNbased CSCM process. The CSCM process was developed as shown in Table I to resolve the redundancy recognition problems and to automate construction supply chain management in and RFID/WSN environment.

\section{IM}

The IM(Intelligent Mover) can identify material sets that are the information medium of RFID/WSN-based CSCM processes. Using ZigBee communication, it can also identify materials that are approaching the IT(Intelligent Trailer) to be loaded and can transmit the identified IM information. Figure III shows an IM prototype and its hardware components.

With regard to the hardware components needed to implement such functions, IM was equipped with a device into which a pallet for loading a material set was inserted. In addition, a load cell was attached to the part of the surface that comes in contact with a pallet to turn the power on or off based on the detected weight as an efficient power supply and an indicator was attached to the IM to allow the operators to visually identify weight deviations. To automatically identify the information on the RFID tag attached to the loaded material sets, an RFID antenna, an RFID reader, and an inverter (used to supply power to the above-mentioned equipment) were configured. Moreover, the operator used an ultra-mobile personal computer (UMPC) as a display and a controller to visually check the automatically identified information. ZigBee was used as the WSN technology to transmit the identified information between equipment. WiBro was also used to support the wireless communication between the IM and the CMS.

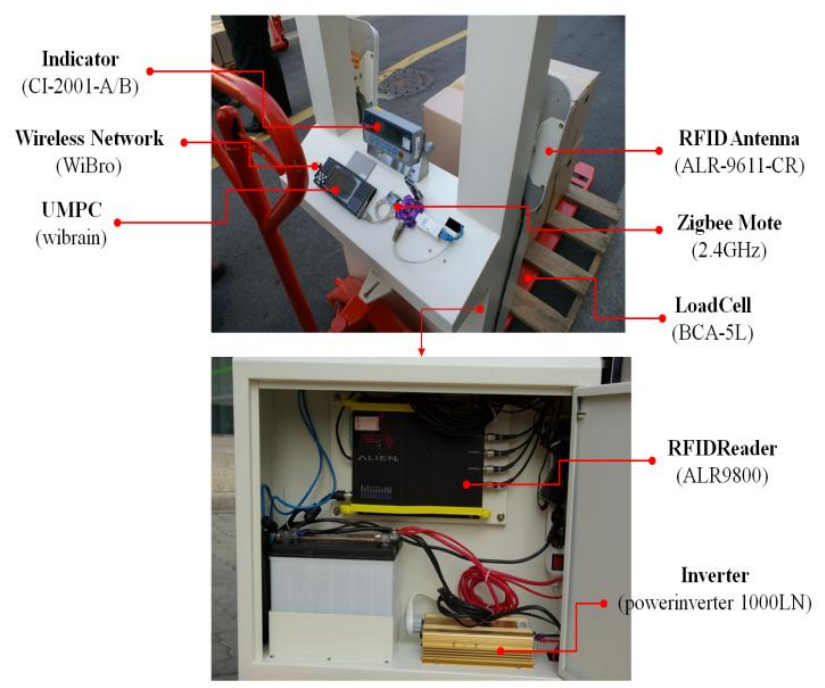

FIGURE III

AN IM PROTOTYPE

The Table II shows the main parts of the RFID/WSNbased equipment and the specification of them. 
TABLE II

MAIN PARTS OF THE RFID/WSN-BASED EQUIPMENT

\begin{tabular}{|c|c|}
\hline Equipment & Specification \\
\hline $\begin{array}{l}\text { RFID tag } \\
\text { (ALN 9654) }\end{array}$ & $\begin{array}{l}\text { EPCglobal Class1 Gen2/ISO/IEC 18000-6C } \\
\text { Intergrated circuit: Alien Higgs-3 } \\
\text { Operating frequency: } 840-960 \mathrm{MHz}\end{array}$ \\
\hline $\begin{array}{c}\text { RFID } \\
\text { Reader } \\
(\text { ALR-9800) }\end{array}$ & $\begin{array}{l}\text { Architecture: Xscale processor, Linux, } 64 \text { Mbytes RAM, } 32 \\
\text { Mbytes Flash } \\
\text { EPCglobal Gen2/ISO/IEC 18000-6C } \\
\text { Reader Protocols: Alien Reader Protocal, SNMP, firmware } \\
\text { upgradeable; LAN Protocols: DHCP, TCP/IT, NTP/Frequency: } \\
\text { 902.75-927.25 MHz } \\
\text { Channels: } 50 \text {; channel spacing: } 500 \mathrm{KHz} \\
\text { RF power: Max } 4 \text { watts EIRP Alien Antenna }\end{array}$ \\
\hline $\begin{array}{c}\text { RFID } \\
\text { Antenna } \\
(\text { ALR-9611- } \\
\text { CR) }\end{array}$ & $\begin{array}{l}\text { Frequency: } 890-930 \mathrm{MHz} \\
\text { Polarization: circular; gain: } 6 \mathrm{dBi} \max \\
\text { Beam Width: } 40 \text { degrees nominal } \\
\text { Cross Polarization Rejection : } 20 \mathrm{~dB} \text {, min } \\
\text { Input Impedance: } 50 \text { ohm nominal } \\
\text { Return Loss: }-15 \mathrm{~dB} \text { across frequency range } \\
\text { Cable: LMR-195, } 50 \text { ohm coaxial, reverse polarity } \mathrm{TNC} \\
\text { connector }\end{array}$ \\
\hline $\begin{array}{l}\text { PDA } \\
\text { (ATRF } \\
\text { AT570) }\end{array}$ & $\begin{array}{l}\text { EPCglobal Class1 Gen2 } \\
\text { ISO/IEC 18000-6C /Frequency: } 866-956 \mathrm{MHz} \text {; reading distance: } \\
4 \mathrm{~m} \\
\text { Communication: WLAN, Bluetooth, USB, IrDA1.1., RS-232C } \\
\text { RF power: 1W EIRP }\end{array}$ \\
\hline $\begin{array}{l}\text { UMPC } \\
\text { (WBN- } \\
\text { B12AN3) }\end{array}$ & $\begin{array}{l}\text { Architecture: Windows XP Home } \\
\text { CPU/Chipset: VIA C7M ULV } 1.2 \mathrm{GHz}+\mathrm{VX} 700(3 \mathrm{D} \text { support) } \\
\text { LCD: } 4.8 \text { " WSVGA }(1024 \times 600) \text { touch screen } \\
\text { Memory: } 512 \mathrm{MB} / 1 \mathrm{~GB} \text { DDR2 } \\
\text { Communication: } 802.11 \mathrm{~b} / \mathrm{g} \text { WLAN, Bluetooth } \\
\text { Battery: } 4-C e l 1 / 6-C e l l \\
\text { Weight: } 526 \mathrm{~g}\end{array}$ \\
\hline $\begin{array}{l}\text { ZigBee } \\
\text { Node }\end{array}$ & $\begin{array}{l}\text { TelosB compatible } \\
\text { Interface: USB } \\
\text { Data transfer rate: } 250 \mathrm{Kbps} \\
\text { Frequency: } 2.4 \mathrm{GHz} \text {; recognition distance: } 30 \mathrm{~m} \text {; size: } 40 \mathrm{~mm} \times \\
22 \mathrm{~mm} \times 8 \mathrm{~mm}\end{array}$ \\
\hline
\end{tabular}

\section{IT}

Once the material sets that have been moved by the IM were loaded onto a trailer, the IM transmitted the information regarding the loaded material sets to the trailer through inter-equipment communication. For this function, was developed an IT(Intelligent Trailer) toolkit that can be plugged into a trailer.

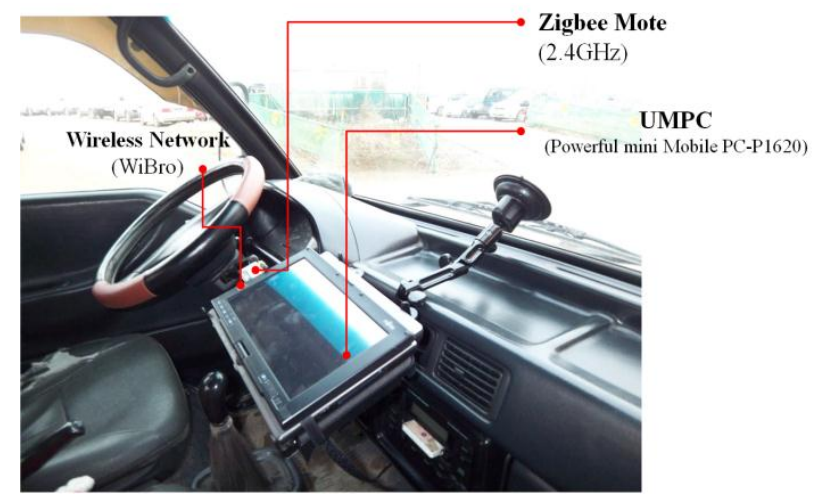

FIGURE IV

AN IT PROTOTYPE
Figure IV shows a prototype of the developed and attached IT. The hardware was configured to be removable, and the UMPC was attached in such a way to allow the trailer operator to check the displayed information regarding the material sets loaded onto the trailer. Similarly to IM, ZigBee technology was configured to support the communication with the IM and WiBro was adopted to communicate with the CMS.

\section{E. GateSensor}

The GateSensor developed in this research is used to monitor and manage the material information flow at both a manufacturing site and a construction site. Specifically, it monitors and controls the passage of material by the IT. When the IT with loaded material approaches the GateSensor, the ZigBee node embedded within the IT recognizes the GateSensor, the RFID reader of which reads the IT tag. The material and vehicle access information is sent to the CSCM server via Wi-Fi or another communication protocol such as code division multiple access or WiBro. Based on the information, the server can control the access of vehicle remotely using GateSensor. The figure V shows A GS prototype and its hardware components.

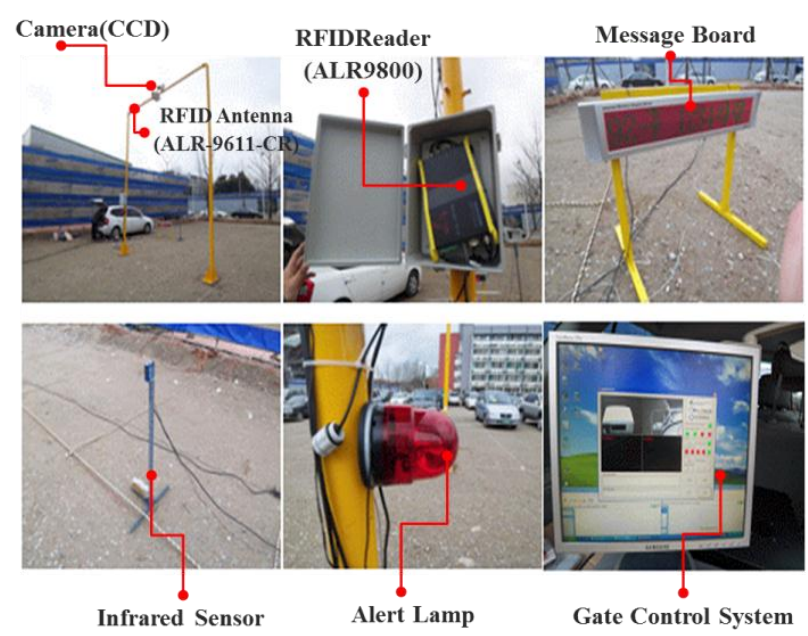

FIGURE V

A GS PROTOTYPE

\section{F. IH}

The IH(Intelligent Hoist) recognizes the IM and materials and lifts them to the planned floor. As shown in figure VI, a toolkit is developed and mounted to a traditional hoist to make create the IH. The toolkit includes an RFID reader, a ZigBee node, Wi-Fi, a touch screen, and a personal computer. The RFID reader of the IH recognizes the RFID tags attached to the materials and communicates with the IM through the ZigBee nodes installed within the IH and IM. The information about the materials and IM that are lifted by the IH is sent to the CSCM server to notify the material flow by the IH and can be confirmed using a PC. IH details are described in Cho et al. (2011). 

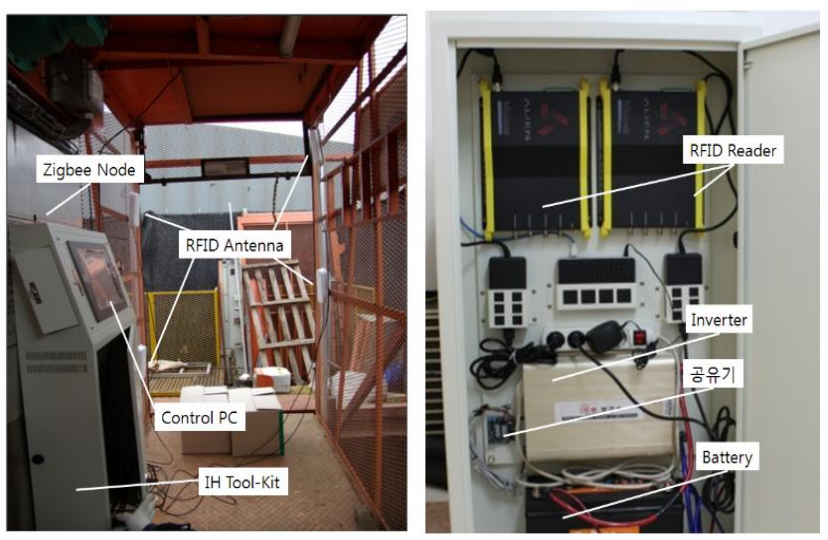

FIGURE VI

AN IH PROTOTYPE

\section{G. Test and Validation}

A test was performed at a construction site in Hwaseong, Korea, to verify and validate the RFID/WSNbased CSCM process developed in this research. The details of the test process are described in Shin et al. (2011), which is part of this research that focuses on the feasibility of SOA applications.

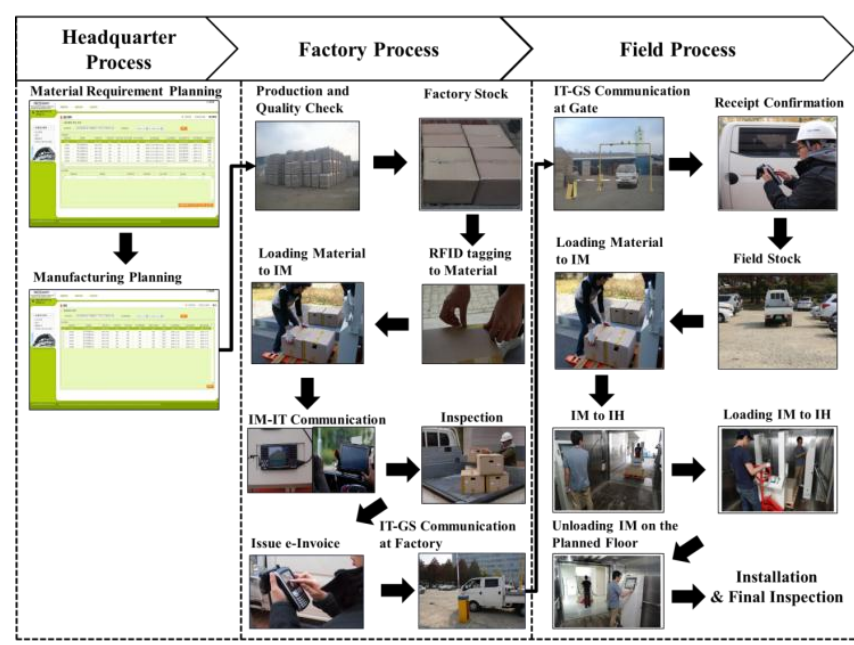

FIGURE VII

TEST PROCESS

Using the test process shown in Figure VII, the test validated that the IM, IT, GateSensor, IH, and CSCM server developed in this research could communicate and share material and equipment flow information during the test.

The test result was analyzed to validate the benefits of the proposed process by comparing time and manpower consumption of the proposed process with conventional 'as-is' process. As described in Shin et al. (2011), the 'asis' process took an excessive amount of time due to the fact that the RFID tags of individual items needed to be read. The overall time was proportional to the number of items to be inspected. On the other hand, our proposed process benefited from the WSN-based communication.
There was an approximate 32\% time reduction in the RFID/WSN-based CSCM process (as-is process: 131 min. vs. proposed process: $89.5 \mathrm{~min}$.).

Furthermore, it was observed that the reliability of the supply chain information can be improved via an automated material flow recognition process. In the proposed process, construction managers can eliminate the need for visual inspection of incoming materials to a site, and the confirmation of material delivery and vehicle access that was typically done through phone calls becomes unnecessary due to intelligent equipment and CSCM server communications.

\section{ISSUES IN RFID/WSN ENVIRONMENT}

Despite the benefits of the process proposed in this research, several issues were found or recognized. The authors believe that the following issues need further research.

\section{A. Reading Performance Issue of RFID}

In this study, it was assumed that the RFID antennas of the IM performed as indicated by their specifications and could identify all of the materials loaded onto the IM within the specified range. Moreover, as shown in Figure VIII, if the identification range of the WSN-based equipment was limited to the equipment size, then the loaded material sets may not have all been identified depending on the material set configuration. As such, an extended range should be allowed, which may cause structural redundancy identification. The focus of this study was how to address redundancy identification as opposed to identification performance and range.
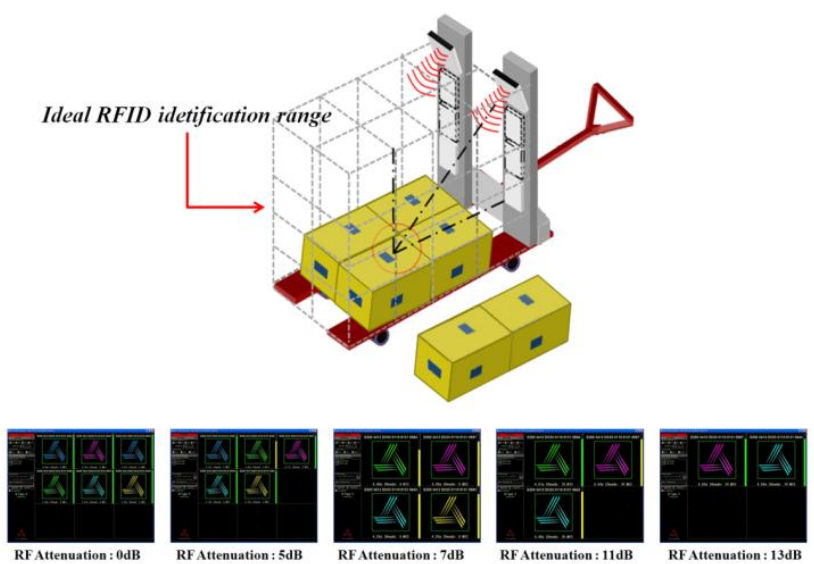

FIGURE VIII

RFID/WSN-BASED MODULE IDENTIFICATION RANGE CONFIGURATION

\section{B. logistics Information Management Issue in Heterogeneous Environments}

The CSCM process proposed in this research features supply chain information that is collected and shared through relationships among various actors, such as human beings, materials, RFID tags, WSN technology, 


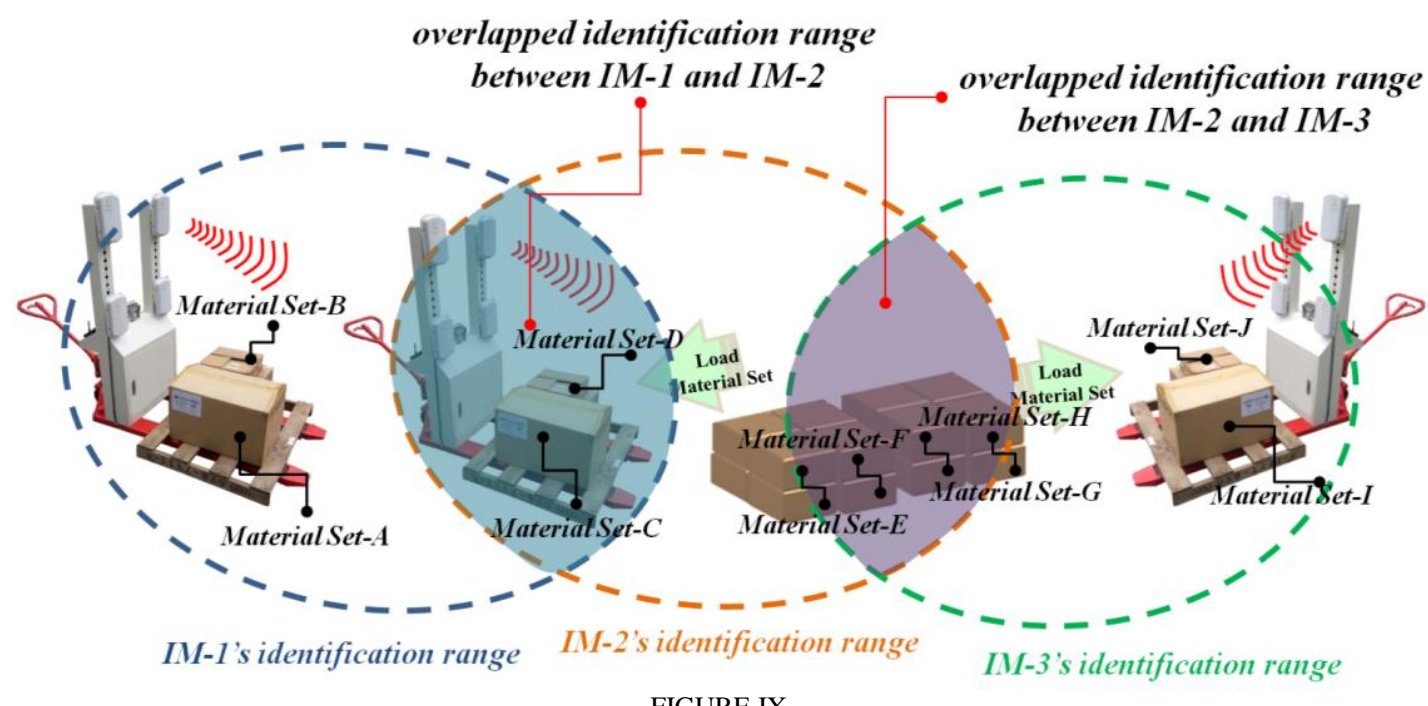

EXAMPLE REDUNDANCY IDENTIFICATION PROBLEM

legacy systems, and equipment, which can be presented as a heterogeneous environment in which an information collect/manage/share supply chain-related information. Based on the need for this framework, the SOA was adopted to support effective and efficient information management in the logistics industry (Li et al., 2006; Xinyan, 2006; Jin et al., 2008). The authors of this paper have since developed a SOA- and RFID/WSN-based information framework (Shin et al. 2011). The proposed framework was adopted during the testing of this research to support information management in heterogeneous CSCM environments.

\section{Redundant Identification Issue}

This paper discusses the redundancy identification problem that may arise during the building of an automated system for CSCM using RFID/WSN technologies. The redundancy identification problem discussed in this paper (Figure IX) focuses on the following: 1) the case in which intelligent equipment recognizes an RFID tag that is not loaded on the equipment but is located within the RFID reader's recognition area, and 2) the case in which the RFID tag is simultaneously read by more than one piece of intelligent equipment.

This redundancy identification problem must be addressed in the implementation of an RFID/WSN-based supply chain because it can cause significant errors in information flow over the supply chain. This issue was initially addressed by Ergen et al. (2007) and Song et al. (2007). Ergen et al. (2007) showed the combination use of RFID and a global positioning system could improve object localization. Song et al. (2007) proposed that in the case that the RFID tagged objects are located at regular intervals such as in a precast stockyard, multiple simultaneous tag readings could be resolved by RF power adjustment. However, the existing research suggests that such a solution could be effective only when the target objects are at a fixed location or move through a predefined path.
The CSCM environment is quite dynamic and uncertain in a way that various actors are not always moving by plan. This research does not include all possible solutions for this, but a workaround was adopted in the test. Redundancy identification can be detected by a CSCM server since it knows which equipment is currently transporting which materials. When the problem is recognized by the server, it notifies an IM manager and provides a list of materials then the manager can correct by mapping the correct material information to the correct IM. Although this research developed the recognition algorithm for redundancy identification, further research is needed regarding the automatic resolution method that does not require human intervention.

\section{CONCLUSIONS}

This research proposes that equipment related to the supply chain process, such as movers, trailers, hoists, and gates, can become main actors in the process via integration with RFID and WSN technologies to create more effective and efficient construction supply chain processes. Material flow is recognized by RFID and ZigBee nodes, and the flow information is communicated in real time through WSN. This research recognized that a redundancy identification problem can occur due to the current limitations of RFID/USN technology, the proposed process removes the reading procedures required at each step of the construction supply chain process and manual collection of the material flow.

An RFID/WSN-based CSCM process was proposed with various actors such as IM, IT, GateSensor, and IH, and the process was verified and validated in a pilot test. The test result showed the benefits and savings in process time as well as effective and efficient information management of CSCM.

The authors believe that this research envisions the future of the CSCM process using state-of-the-art technologies such as RFID and WSN. Further research issues (described chapter $\mathrm{V}$ : reading performance, 
heterogeneous environments, and redundant identification) were identified as well, and this information will hopefully invoke future relevant research.

\section{ACKNOWLEDGEMENTS}

This work was supported by the Korean Institute of Construction \& Transportation Technology Evaluation and Planning (KICTEP; program number "06-Unified and Advanced Construction Technology Program-D16"). It was also supported by the Brain Korea 21 project conducted by Sungkyunkwan University.

\section{REFERENCES}

[1] A. Behzadan, Z. Aziz, C. Anumba, V. Kamat, "Ubiquitous location tracking for context-specific information delivery on construction sites", Automation in Construction, vol. 17, no. 6, pp. 737-748, 2008.

[2] C. Cho, S. Kwon, T. Shin, S. Chin, Y. Kim, "A development of next generation intelligent construction liftcar toolkit for vertical material movement management", Automation in Construction, vol. 20, no. 1, pp. 14-27, 2011.

[3] E. Ergen, B. Akinci, R. Sacks, "Life-cycle data management of engineered-to-order components using radio frequency identification", Advanced Engineering Informatics, vol. 21, no. 4, pp. 356-366, 2007.

[4] E. Jaselskis, M. Anderson, C. Jahren, Y. Rodriguez, S. Njos, "Radio-Frequency Recognition Application in the Construction Industry", Journal of Construction Engineering and Management, ASCE, vol. 121, no. 2, pp. 189-196, 1995.

[5] E. Jaselskis, T. El-Misalami, "Implementing Radio Frequency Recognition in the Construction Process", Journal of Construction Engineering and Management, ASCE, vol. 129, no. 6, pp. 680688, 2003.

[6] E. William, J. Kirby, L. Liu, "Verification and validation of a project collaboration tool", Automation in Construction, vol. 17, no. 2, pp. 201-214, 2008

[7] G. Zhang, D. Tao, "Research on the Application of Zigbee Wireless Sensor Network in the Inter-Vehicle Communication System", International Conference on Transportation Engineering, pp. 26142619, 2007.

[8] H. Hiremath, M. Skibniewski, "Object-oriented modeling of construction processes by unified modeling language", Automation in Construction, vol. 13, no. 4, pp. 447-468, 2004.

[9] H. Khoury, V. Kamat, "Evaluation of position tracking technologies for user localization in indoor construction environments", Automation in Construction, vol. 18, no.4, pp. 444-457, 2009.

[10] I. Akyildiz, W. Su, Y. Sankarasubramaniam, E. Cayirci, "Wireless sensor networks: a survey", Computer Networks, vol. 38, no. 4, pp. 393-422, 2002.

[11] J. Han, S. Kwon, M. Cho, "Development of Material Management System and Field Tests Using RFID Technology on High-Rise Building Construction", Journal of the Architectural Institute of Korea (Structure \& Construction Section), vol. 22, no. 10, pp. 121-128, 2006.

[12] J. Song, C. Caldas, E. Ergen, C. Haas, B. Akinci, "Field Trials of RFID Technology For Tracking Pre-fabricated Pipe Spools", Proceedings of the 21st International Symposium on Automation and Robotics in Construction, pp. 664-669, 2004.

[13] J. Song, C. Hass, C. Caldas, E. Ergen, B. Akinci, “Automating the task of tracking the delivery and receipt of fabricated pipe spools in industrial projects", Automation in Construction, vol. 15, no. 2, pp. 166-177, 2006.

[14] L. Haifeng, Z. Yan, C. Lei, "Design of Wireless Logistics Pallet Location System Based on ZigBee Protocol", The Eighth International Conference of Chinese Logistics and Transportation Professionals, pp. 2115-2120, 2008.

[15] L. Xinyan, "Using SOA to design a public logistics service platform", Computer systems and applications Journal, vol. 11, no. pp. 33-37, 2006.

[16] M. Goodrum, A. McLaren, A. Durfee, "The application of active radio frequency identification technology for tool tracking on construction job sites", Automation in Construction, vol. 15, no. 3, pp. 292-302, 2006.

[17] M. Skibniewski, W. Jang, "Localization Technique for Automated Tracking of Construction Materials Utilizing Combined RF and Ultrasound Sensor Interfaces", The Proceedings of the ASCE International Workshop on Computing in Civil Engineering, July 24-27, Pittsburghm PA, USA, pp. 657-664, 2007.

[18] N. Hoult, R. Paul, A. Fidler, P. Hill, C. Middleton, "Long-Term Wireless Structural Health Monitoring of the Ferriby Road Bridge", Journal of Bridge Engineering, vol. 15, no. 2, pp. 153159, 2010.

[19] S. Chin, S. Yoon, C. Choi, C. Cho, "RFID+4D CAD for Progress Management of Structural Steel Works in High-Rise Buildings", Journal of Computing in Civil Engineering, vol. 22, no. 2, pp. 7489, 2008.

[20] S. Chin, S. Yoon, C. Choi, S. Kwon, Y. Kim, C. Kim, "Lessonslearned from RFID Applications in the Korean Construction Industry", Construction Research Congress, ASCE/CIB, pp. 166173, 2007.

[21] S. Lee, N. Park, C. Cho, H. Lee, S. Ryu, "The Wireless Broadband (WiBro) System for Broadband Wireless Internet Services", IEEE Communications Magazine, pp.106-112, 2006.

[22] S. Yoon, J. Song, T. Shin, S. Chin, "A GateSensor for Effective and Efficient Entering/Taking Management of Vehicles for Construction Logistics", Korean Journal of Construction Engineering and Management, vol. 12, no. 1, pp. 85-96, 2011.

[23] S. Yoon, S. Chin, Y. Choi, C. Choi, "An RFID-Based Supply Chain Management System for Curtain Walls", CITC-III Advancing Engineering, Management and Technology, Athens, Greece, pp. 15-17, 2005.

[24] T. Shin, S. Chin, S. Yoon, S. Kwon, “A service-oriented integrated information framework for RFID/WSN-based intelligent construction supply chain management", Automation in Construction, vol. 20, no. 6, pp. 706-715, 2011.

[25] W. Andrew, "Commercial applications of wireless sensor networks using ZigBee”, IEEE Communications Magazine, vol. 45, no.4, pp.70-77, 2007.

[26] W. Jang, W. Healy, M. Skibniewski, "Wireless sensor networks as part of a web-based building environmental monitoring system", Automation in Construction, vol. 17, no. 6, pp. 729-736, 2008.

[27] W. Wu, H. Yang, D. Chew, S. Yang, A. Gibb, Q. Li, “Towards an autonomous real-time tracking system of near-miss accidents on construction sites", Automation in Construction, vol. 19, no. 2, pp. 134-141, 2010.

[28] Y. Cho, J. Youn, D. Martinez, "Error modeling for an untethered ultra-wideband system for construction indoor asset tracking", Automation in Construction, vol. 19, no. 1, pp. 43-54, 2010.

[29] Y. Guizhen, C. Lianyu, D. Nenggen, "The ZigBee Application on Intelligent Bus Dispatch", International Conference on Transportation Engineering, pp. 2506-2511, 2007.

[30] Y. Li, B. Dong, "Supply Chain Information Sharing System Based on SOA", Logistics technology Journal, vol.7, pp. 217-220, 2006.

[31] Z. HaiJin, L. WenJu, K. Zhen, "Supply Chain Simulation: Collaborative Design System based on SOA - A Case Study in Logistics Industry _", Proceedings Of World Academy Of Science, Engineering and Technology, vol. 39, pp. 364-367, 2008. 